\title{
Venous gas embolism as a predictive tool for improving CNS decompression safety
}

\author{
A. Møllerløkken · S. E. Gaustad • M. B. Havnes · \\ C. R. Gutvik · A. Hjelde · U. Wisløff · A. O. Brubakk
}

Received: 27 January 2011 / Accepted: 2 May 2011 / Published online: 19 May 2011

(C) The Author(s) 2011. This article is published with open access at Springerlink.com

\begin{abstract}
A key process in the pathophysiological steps leading to decompression sickness (DCS) is the formation of inert gas bubbles. The adverse effects of decompression are still not fully understood, but it seems reasonable to suggest that the formation of venous gas emboli (VGE) and their effects on the endothelium may be the central mechanism leading to central nervous system (CNS) damage. Hence, VGE might also have impact on the long-term health effects of diving. In the present review, we highlight the findings from our laboratory related to the hypothesis that VGE formation is the main mechanism behind serious decompression injuries. In recent studies, we have determined the impact of VGE on endothelial function in both laboratory animals and in humans. We observed that the damage to the endothelium due to VGE was dose dependent, and that the amount of VGE can be affected both by aerobic exercise and exogenous nitric oxide (NO) intervention prior to a dive. We observed that NO reduced VGE during decompression, and pharmacological blocking of NO production increased VGE formation following a dive. The importance of micro-nuclei for the formation of VGE and how it can be possible to manipulate the formation of VGE are discussed together with the effects of VGE on the organism. In the last part of the review we introduce our
\end{abstract}

Communicated by Dag Linnarsson.

A. Møllerløkken $(\varangle) \cdot$ S. E. Gaustad · M. B. Havnes .

C. R. Gutvik · A. Hjelde · U. Wisløff · A. O. Brubakk

Baromedical and Environmental Physiology Group,

Department of Circulation and Medical Imaging,

Norwegian University of Science and Technology,

Medisinsk teknisk forskningssenter,

Olav Kyrres gt 9, 7489 Trondheim, Norway

e-mail: andreas.mollerlokken@ntnu.no thoughts for the future, and how the enigma of DCS should be approached.

Keywords VGE $\cdot$ Endothelium $\cdot$ Decompression sickness · Decompression stress

\section{Introduction}

Decompression sickness (DCS) is caused by gas coming out of the solution, a phenomena that normally requires supersaturation of inert gases in the organism. However, numerous experiments indicate that bubbles originate as pre-existing gas filled nuclei (Yount 1988; Blatteau et al. 2006) and more attention has lately been given to the importance of these nuclei. Their presence and characteristics are believed to be just as important for the occurrence of DCS as supersaturation, and has given a foundation for novel approaches to prevent DCS.

Signs and symptoms of DCS differ with the pressure profile and the mixture in the breathing gas, but the common first step is the formation of gas bubbles. The emerging evidence of the effects of damage inflicted to the endothelium by venous gas embolism (VGE) has led us to the hypothesis that VGE evolution is the main mechanism for neurologic DCS (CNS DCS) through its adverse effects on the CNS.

For a long time, researchers have suggested that formation of gas bubbles may be closely related to the susceptibility of DCS. Boyle observed bubbles leading to the development of symptoms of illness in 1659 (Boyle 1670). In line with this, Paul Bert discovered that DCS was related to nitrogen bubbles, and observed that recompression treatment relieved the pain associated with these bubbles (Bert 1943). Throughout the nineteenth century, the pathophysiology of DCS was studied, but little was done on how to 
prevent injury. Then, in 1908 Haldane et al. (Boycott et al. 1908) revolutionized the safety of diving with the publication of the first true decompression tables (Lippmann and Mitchell 2005). Today, 100 years after Haldane, the enigma of DCS is still not resolved. Therefore, the ability to take preventive and therapeutic advantage of the understanding of the mechanisms behind DCS has not lost its importance.

In-depth studies of extreme environmental physiology, such as that experienced during diving and decompression requires methods with a wide system-biological approach. There are obvious ethical concerns related to extreme physiological exposures of humans. In addition it seems unlikely that one can obtain tissue to study cellular and molecular mechanisms of DCS from organs such as the brain, heart and arteries in man. Therefore, we have to rely upon appropriate animal models to improve our understanding of the underlying causes of DCS. Fortunately, such models exist and we have shown that results from experiments using these models have been translated into clinical studies of DCS (Dujic et al. 2004, 2006a, b). We therefore strongly suggest that in the hunt for the answers to the enigma of DCS, one needs to perform translational research.

\section{Bubbles and risk of DCS}

Bubbles formed during or after an ascent can have their origin in two different mechanisms; either evolving from any dissolved inert gas (most often nitrogen) or they can originate through pulmonary barotrauma. Inert gases are not involved in the manifestation of barotraumas, since barotrauma can be generated from ascents from only 1 to 2 msw. As such, the present review will not focus on the formation of bubbles from barotrauma, but rather on the formation of bubbles from dissolved, inert gas.

If the ambient pressure is reduced sufficiently below the partial pressure of the dissolved gas in the tissue and blood, formation of bubbles can lead to DCS. Gas bubbles may form at any site in the body and can be categorized in two groups; stationary or circulating bubbles. Stationary bubbles are formed in the tissues and may produce local damage and intravascular blockage, which gives rise to a variety of symptoms and signs. While there exists no method to measure stationary bubbles, Doppler and echocardiography are commonly used to measure VGE in the central venous pools. The lungs filter and remove the nitrogen bubbles formed in the veins, whereas arterial bubbles can block and/or damage the blood vessels in vital organs such as the brain and heart. Thus it seems that venous bubbles are less dangerous compared to arterial bubbles. However, venous bubbles can become arterial bubbles through shunts in the lungs and/or the heart. Recent studies have reported high incidences of VGE with subsequent arterialization after trimix diving and after no-decompression air dives (Ljubkovic et al. 2010a, b). Both the studies with trimix dives and with no-decompression dives used standardized protocols without any violations, and without any clinical signs of adverse effects of decompression. These two recent studies have again raised the question of possible long-term adverse effects of diving due to the unknown effect of repeated exposures to arterialization of VGE.

Traditionally, DCS is divided into type I or type II, depending on the symptoms and manifestations. Type I concerns only the symptoms of musculoskeletal pain, while type II are the more serious manifestations mainly related to the CNS. It has been suggested that there has been a shift from type I to type II DCS in the recent past, as type II is now more common (Francis and Mitchell 2003). In as many as $77 \%$ of the cases of DCS, the UK Institute of Naval Medicine showed prevalence of neurological manifestations. Limb pain was found in $49 \%$ of the cases. The shift in manifestation may be explained by the introduction of dive computers, leading to increased bottom time and deeper dives (Andric et al. 2003). On the other hand, an alternative explanation is the increased focus on neurological damage to divers.

Involvement of VGE in CNS DCS has been discussed for several years. Clinical observations showing that the spinal cord is more involved in DCS compared to the brain made Hallenbeck et al. (1975) suggest that gas embolism is not a mechanism for spinal cord DCS. If VGE is the main mechanism, one would rather suspect higher perfused organs to be more involved in CNS DCS than actually observed in the clinical setting. However, it is well documented by using ultrasound contrast media that small gas bubbles will lodge in small vessels and that the distribution of these bubbles is critically dependent on size (Talu et al. 2007; Klibanov 2006). Other studies have shown that vascular gas bubbles can be located in the spinal cord in DCS (Sparacia et al. 1997). Several investigators have shown that the brain might be more involved in CNS DCS than previously believed. Moon et al. (1989) showed by contrast echocardiography that gas emboli play an important role in CNS DCS. They observed a higher incidence of atrial septal defects in cases with neurologic DCS. Such defects would allow VGE to be transported from the right to the left side of the circulation.

Involvement of VGE in neurologic DCS has been showed both in animals and in men (Wilmshurst et al. 1994; Vik et al. 1993; Germonpre et al. 1998). However, several other researchers are not convinced about this role of VGE in relation to DCS (Smith et al. 1990; Cross et al. 1992; Langton 1996). In addition to the blood flow argument cited above, the observation of a patent foramen ovale (PFO) in approximately $30 \%$ of the population does 
not fit with the numbers developing DCS. The relevance of shunting is questioned since many divers with detectable shunts have no history of DCS (Wilmshurst et al. 1989; Cross et al. 1992). On the other hand, Wilmshurst and Bryson (2000) showed that individuals with right to left shunting had higher risk of neurological decompression illness than individuals without shunts. They also pointed out that individuals with smaller shunts would have intermediate risk, but other factors like individual susceptibility to venous bubble formation would affect the risk as well. Another implication for divers with a PFO to take into consideration is the change in intrathoracic pressure that takes place during a Valsalva maneuver (Balestra et al. 1998). A Valsalva is used during descent to equalize pressure in the middle ear with the ambient pressure, and can cause an increase in right arterial pressure which will open a PFO (if present). Balestra et al. concluded that movements other than the Valsalva also can increase the intrathoracic pressure, such as sustained isometric exercise or abdominal strains. These other maneuvers were more likely to cause post-release central blood shifts, which made them recommend that divers with PFO should avoid any exercise that is likely to increase the intrathoracic pressure for prolonged periods of time. A more recent study also showed that over a period of seven years the permeability of PFO in a group of sports divers had changed (Germonpre et al. 2005). For the first time, an increase in PFO size was documented in humans, an increase that might imply that divers could develop more susceptibility for serious DCS over time. Additionally, an important point made by Wilmshurst and Bryson was that CNS DCS could also be related to lung disease and unsafe dive profiles, not only to bubbles shunting from the venous to the arterial side. Symptoms from the joints and muscles were not correlated with shunts.

\section{Adverse effects of VGE}

VGE does not necessarily lead to DCS, but VGE is an indicator of decompression stress. The relationship between decompression stress and DCS is not straight forward; there is no positive correlation. However, since there are no other measurable indications for mechanisms in relation to VGE and decompression stress, the measurement of VGE could be established as the standard for validating decompression procedures.

VGE can be seen in large amounts without any commensurate manifestation of decompression injury (Nishi et al. 2003). However, the risk of DCS increases with the amount of bubbles detected (Eftedal et al. 2007a). In his masters thesis, Sawatzky (1991) systematically analyzed maximum Doppler scores and DCS incidence in a total of 3,234 dives.
He found only one incidence of DCS not accompanied by detectable bubbles, giving a negative predicting value of 0.999 and a sensitivity of detectable bubbles as a test for DCS of 0.99. However, Sawatzky also detected bubbles without any symptoms of DCS, which gave a positive predictive value of only 0.04 . This illustrates that having detectable bubbles yields only a $4 \%$ chance of developing DCS. Even when using high bubble grades as test criterion, the positive predictive value is low (Eftedal 2007). As Eftedal (2007) stated in his doctoral thesis: ".. the absence of detectable bubbles is a good indicator of decompression safety, but the occurrence of bubbles, even high grades, is a poor predictor of decompression sickness". Since there seems to be only a statistical relationship between VGE and DCS, the detection of bubbles by ultrasound can not be used as diagnostic criteria for DCS. Arterial bubbles are often connected to the more serious cases of decompression injuries (Neuman 2003), which indicates that VGE is a relevant stress indicator for such symptoms; thus quantification of bubbles in the venous system can offer a graded measure of decompression safety (Nishi et al. 2003; Eftedal et al. 2007a, b). VGE gives only a fraction of the total picture of what is happening in the body, but is significant as it is the only objective and quantitative measurement available to evaluate decompression stress.

\section{VGE alter the endothelial function and changes barrier permeability}

The endothelium is activated by sensing various physiologic stimuli and triggering release of multiple vasoactive substances, including nitric oxide (NO). Such physiologic stimuli can be both substances present in the blood or the shear stress associated with the blood flow (Shaul 2002). A large number of vasoactive substances are produced and secreted from endothelial cells to act on the underlying vascular smooth muscle cells. The balance between dilating and contracting factors is critical for maintaining vascular homeostasis (Cockcroft 2005). As such, the endothelium plays a key role in the short- and long-term regulation of the cardiovascular system and is the source of many factors that influence blood flow, blood coagulation as well as angiogenesis (Triggle et al. 2003). Several studies have shown reduced endothelial function after exposure to vascular bubbles (Nossum et al. 1999, 2002; Brubakk et al. 2005), suggesting that this effect may be a central mechanism in the development of serious decompression injury, and possibly also in long-term injury (Brubakk et al. 2007). A more recent study by Obad et al. (2010) reported endothelial dysfunction after successive trimix dives and at the same time also augmented generation of reactive oxygen species (ROS). 
Even in the absence of clinical manifestations, VGE can cause changes in barrier permeability. Proteins and leukocytes can be allowed into the extravascular brain tissue with subsequent formation of edema if the bloodbrain- and/or blood-lung barrier (BBB and/or BLB) is broken down (Hjelde et al. 2002; Chryssanthou et al. 1977; van Hulst et al. 2003). Polymorphonuclear leukocytes (PMNs) play a central role in the bubble-induced inflammatory response (Martin and Thom 2002; Nyquist et al. 2004). Nossum et al. (2003) demonstrated reduced PMN infiltration in rabbits given anti-C5a monoclonal antibody and thus protected the endothelium against injury caused by small amounts of bubbles. From animal models of gas embolism, leukocytes have been implicated in the progressive fall in cerebral blood flow and decreased cerebral function (Dutka et al. 1989; Helps and Gorman 1991). PMN accumulation in rat brain is also demonstrated to be a part of an acute inflammatory response to brain injury (Clark et al. 1996; Schoettle et al. 1990). Schoettle et al. (1990) found a significant correlation between PMN accumulation and the development of cerebral edema. Similarly, leukopenic dogs suffered less microcirculatory damage after injection of air into the internal carotid artery than did control dogs (Dutka et al. 1989). In a recent study by Thom et al. (2011), circulating microparticles (MPs) were found to be elevated by decompression stress. Decompression from greater pressures resulted in higher amounts of MPs from different cell types, and the study gave a pathophysiological link between MPs and tissue injuries. Further, in a study by (Glavas et al. 2008) a possible link between formation of VGE after a single dive and impaired arterial vasodilatation were found. Together with the previously mentioned work, this points toward the hypothesis that DCS is an inflammatory disease.

The symptomatology of DCS is heterogeneous and not entirely explained by a mechanically induced tissue injury. The bubbles may compress against the endothelial wall, obstructing and stripping the endothelial cells (Nossum et al. 1999). However, it is quite possible that the pathogenesis of DCS, at least in part, may be of an inflammatory origin. Empirically, there is an interindividual susceptibility to DCS and it has been suggested that individual differences in inflammatory response might be a part of this mechanism (Ward et al. 1987). Platelets and leukocytes have been found to aggregate and adhere to intravascular bubbles, thus representing a foreign surface that may induce biochemical changes, which was discussed early in the 1970s (Philp et al. 1972). A subsequent inflammatory activation has been demonstrated by several studies (Ward et al. 1987; Ersson et al. 2002; Bergh et al. 1993; Brenner et al. 1999; Boussuges et al. 1998).

\section{Nitric oxide}

Our studies have shown that Nitric oxide (NO) may play a significant role in the formation of vascular bubbles (Wisloff et al. 2003, 2004; Mollerlokken et al. 2006; Dujic et al. 2006b) and that both exercise and NO protects against vascular bubble formation and DCS (Dujic et al. 2004, 2006b; Wisloff et al. 2004). Further, exercise is shown to induce production of eNOS in the vascular system through increased blood flow and shear stress (Green et al. 2002) (Kingwell et al. 1997). Why NO has this positive effect protecting animals and humans from bubble formation during decompression, may not only be attributed to its effect as a potent vasodilator, but it also reduces the adhesiveness of cellular particles to the endothelial surface (Kerem and Mehmet 2000). Since both factors may influence the surface properties of the endothelium, this may reduce the number of nuclei available for bubble production.

NO is a free radical gas generated by NO synthase (NOS) through oxygenation of one of the guanidine nitrogen atoms of L-Arginine and co factors situated in the blood (Alderton et al. 2001). They are products of different genes, with different localization, regulation, catalytic properties and inhibitor sensitivity (Alderton et al. 2001). The isoforms are referred as neuronal NOS (nNOS or type 1), inducible NOS (iNOS or type 2) and endothelial NOS (eNOS or type 3). In the vascular wall, most of the NO is believed to derive from eNOS, but both iNOS and nNOS are also shown to participate in vascular function (Moncada and Higgs 2006; Kavdia and Popel 2004).

The NO pathway is not exclusive for decompressionrelated bubbles, as impaired endothelial function due to impaired NO production/availability has been observed in a number of cardiovascular diseases (VanWijk et al. 2003). Numerous studies have shown that endothelial dysfunction is independently related to future cardiovascular events (e.g. myocardial infarction, stroke, transient ischemic attack) (Gonzalez and Selwyn 2003).

\section{Manipulation of VGE: safer decompression?}

Traditionally, the reduction of VGE to prevent DCS has been achieved by changing ascent rates. Even though the present procedures have low incidence of DCS, we have demonstrated experimentally (Brubakk et al. 2003) and theoretically (Gutvik and Brubakk 2008) that there is considerable room for improvement. In a number of studies using rats, we have shown that the amount of VGE following a dive can be significantly reduced by performing heavy exercise, $20 \mathrm{~h}$ before the dive (Wisloff et al. 2004). The protective effect disappeared $48 \mathrm{~h}$ later, while exercise closer to the dive had no effect. An increased blood flow will 
increase the shear stress which again increases the endothelial production of NO. The process is not instantaneous, and this might explain the protective effect of exercising $20 \mathrm{~h}$ before the dive. By blocking NO we have previously demonstrated that bubble production will increase, and that the protective effect of exercise can be simulated by adding NO (Dujic et al. 2006a, b; Mollerlokken et al. 2006; Wisloff et al. 2003, 2004). The protective effect of exercise preceding a dive was confirmed in man (Dujic et al. 2004). The beneficial effect of exercise during decompression has also been demonstrated by reducing the number of VGE generated, probably through a NO mechanism (Blatteau et al. 2005; Broome et al. 1995; Dujic et al. 2005). An alternative hypothesis is that increased cardiac output (as a result of the exercise) and hence the tissue blood flow, results in increased gas elimination. Thus, timing and intensity of exercise performed are critical factors, as to whether the effects are harmful or beneficial. As such, exercise may represent a novel way to reduce the risk of DCS. However, it has been shown that bubble production is increased by NO blockage in sedentary but not in exercised rats, indicating that the beneficial effect of exercise may be mediated by other factors than NO (Wisloff et al. 2003). Other pre-dive strategies have also reduced the amount of VGE following decompression. (Blatteau et al. (2008) increased the amount of NO and HSP without exercise, but by a pre-dive session in a sauna. Also pre-dive vibration has been reported to reduce the amount of VGE after a dive, without any changes in the endothelial function, and hence no increase in the amount of NO (Germonpre et al. 2009).

Our laboratory has previously shown that even low bubble loads lead to endothelial dysfunction (Nossum et al. 2002), and that this damage is dose dependent (Nossum et al. 1999), an observation supported by others (Rosenblum 1997). Furthermore, we have shown a close relationship between maximal oxygen uptake (aerobic fitness) and endothelial function (Haram et al. 2006). Exercise is also one of the treatments recommended for chronic endothelial dysfunction, which shows the importance of physical fitness in diving. Since younger, slimmer, or aerobically fitter divers are known to produce less bubbles compared with older, fatter, or poorly physically fit divers (Carturan et al. 2002), personalized decompression tables and computers seem prudent. As Moon et al. (1995) wrote: "the probabilistic models on which tables and computers are based should reflect the individual reality of the divers, to enable them to conduct their dives in accordance with their individual characteristics".

A comprehensive understanding of biochemical, immunological and molecular-biological pathways involved in DCS will improve decompression procedures and the understanding of the basal physiology behind the adverse effects of decompression. Based on previous work, one hypothesis is that microbubbles are influenced by differences in caveolae (see below), where we suggest that bubble nuclei may be located. As with other types of technology and research, the use of animal models is under constant development. Genetically modified animals, in particular mice, give the opportunity to perform studies that might explain the individual differences in relation to the adverse effects of decompression.

\section{Caveolae}

Bubbles need something to grow from, as the negative pressures necessary for spontaneous bubble formation goes far beyond what is feasible in a decompression situation (Zheng et al. 1991). However, bubbles have other characteristics that can help us identifying their origin, one of them being that bubbles are extremely stable on hydrophobic surfaces (Liebermann 1957). Thus, lipid rich micro-domains on the surface of the endothelium can have a particular propensity for the formation and/or stable attachment of bubble nuclei (Chappell and Payne 2005). Brubakk (2004) postulated that hydrophobic sites can exist on the endothelium surface. This further indicates that the caveolae are attractive sites for the formation of bubble nuclei, since reduction in surface tension of hydrophobic membranes have been shown to increase the number of stable nuclei (Gaskins et al. 2001). Caveolae are also the location for eNOS production (Wisloff et al. 2007), which further supports the attractiveness of this specific location for bubble formation. Invaginations in the vascular wall have been suggested to represent such a stabilizing mechanism for bubbles.

\section{Endothelial microparticles}

Activation of the endothelium generates endothelial microparticles (EMP), which are fragments of activated endothelial cells. These may in turn reduce the endothelial function, possibly by increasing expression of the endothelial adhesion molecules such as vascular cell adhesion molecule-1 (VCAM-1), intracellular adhesion molecule-1 (ICAM-1) and E-selectin, and by influencing NO production (Jimenez et al. 2003; Brodsky et al. 2004). Following a decompression, this activation could be caused by endothelial damage from gas bubbles. The EMPs can pass the lung filter and as such directly affect the endothelium dependent vasorelaxation on the arterial side by a mechanism that involves diminished production and/or bioavailability of $\mathrm{NO}$ as shown by Brodsky et al. (2004). Microparticles (MPs) can have other origins besides the endothelium, and circulating MPs have been shown to be increased after simulated scuba 
diving (Vince et al. 2009). Decompression stress has also been reported to elevate the amount of MPs (Thom et al. 2011). The suggested mechanisms behind the elevated MPs are oxidative stress and apoptosis.

Another attractive candidate to improve decompression safety is the use of fluorocarbons. These compounds are characterized by a high gas dissolving capacity $\left(\mathrm{O}_{2}, \mathrm{CO}_{2}\right.$, inert gas), low viscosity, chemically and biologically inert (Spahn 2000). The $\mathrm{O}_{2}$ solubility of fluorocarbons is 20 to 25 times greater than that of blood plasma (Mahon et al. 2006). Thus, intravenous administration of these agents may increase tissue $\mathrm{O}_{2}$ delivery. Also, because of higher diffusion gradients, fluorocarbons would affect bubbles by reducing the bubble size. Reduced mortality following gas embolism (Lutz and Herrmann 1984), reduced brain infarct size (Cochran et al. 1997) and improved cardiovascular function after air embolization (Tuman et al. 1986) have been found in animals, but the efficiency of perfluorocarbons in humans are still to be proven (Moon 2004).

Exposure to different environmental stress conditions leads to the formation of stress proteins commonly known as heat shock proteins (HSP). The HSP are chaperones for protein molecules and protect the organism against injury. However, in some cases, expression of HSP may contribute to injury. Divers may be exposed to a number of stressors that could possibly influence the effects of bubbles. Saturation divers are exposed to considerable stress (hyperoxia, hard physical work, exposure to infections a.s.o.), which could lead to an increase in HSP over longer periods of time. Of particular interest is the exposure to bacteria, as infections are still a significant problem in saturation diving operations (Ahlen et al. 1998). HSP are present in most cells, and play a key role in normal cellular homeostasis and cell protection from damage in response to stressstimuli. Endurance exercise is an example of a stressor which increases the expression of HSP70 (Kregel 2002). Increased expression of HSP70 and subsequent protection of rats from air embolism-induced lung injury has been found with heat shock pre-treatment before diving (Huang et al. 2003). Thus, exercise-induced HSP70 production seems to affect the bubble formation after diving with a mechanism other than the NO pathway (Blatteau et al. 2005). In a study by Montcalm-Smith et al. (2007), certain biomarkers were investigated in order to use these as indicators of DCS. The use of biomarkers has also been investigated by Havnes et al. (2010).

There are two potential sources of bubbles; they can either be formed in blood and/or tissues or they can be introduced into the blood. There is a statistical relationship between VGE and DCS (Sawatzky 1991; Eftedal 2007), and VGE also have the potential to induce long-term effects. As a consequence, both mechanisms of bubble formation and any physiological effects need to be elucidated.
Being a consequence of gas uptake and elimination, a major task for the future is to utilize techniques to measure the amount of gas that is liberated. When venous gas infusion or gas injections have been used to study effects of VGE, the infused volume of gas is known, although the size of the bubbles that enter the pulmonary circulation is unknown. Since the physiological effects of VGE after decompression are dependent on the degree of venous gas loading, it is important to quantify the amount of gas. The ideal model to study mechanisms of bubble formation as well as physiological effects of the bubbles and pathophysiological mechanisms must include a method to measure or estimate the amount of gas that appears as gas bubbles.

\section{Concluding remarks and future perspectives}

Bubbles have been detected on both the venous and arterial side of the circulation following decompression. Even though many questions still exist, the damaging effects of these bubbles on the endothelium are well documented. We suggest that this is a central mechanism in the development of serious DCS, and further that it can be a mechanism for the possible long-term health effects of diving. Protection against DCS has traditionally focused on influencing bubble formation by changing ascent procedures from a dive. Rather we believe that the mechanisms behind DCS should be focused upon, since numerous studies from our laboratory have shown that vascular bubble formation is significantly influenced by the properties of the endothelium. These properties are known to change in various ways and it reveal new approaches to prevent injury from diving.

There is evidence that the occurrence of at least some forms of DCS is not entirely consistent with purely physical processes. As a result, inability to predict or control DCS severity is perhaps the most glaring deficiency of modern DCS prevention algorithms (Tikuisis and Gerth 2003). This is largely due to the relatively mild nature of the DCS cases in the data available for model calibration. Changes in the physical or chemical surrounding of any organism, such as the pressure changes experienced on ascent from diving, are likely to trigger adaptive changes in gene expression patterns. One of the remaining cornerstones in the enigma of DCS is the large variability between individuals, both in DCS incidence and bubble formation. Even in animal experiments, where physiological variables are tightly controlled, there is a considerable and significant difference in response between individuals. The same dive can produce few or many bubbles, and the response to bubbles differs as well. Current knowledge points toward possible genetic link between endothelial dysfunction and DCS (Brubakk et al. 2007). This is based on the observation that some genes known 
to be involved in NO homeostasis display considerable individual difference in activity, which again is known to trigger susceptibility to a number of diseases.

Currently, we at the Baromedical and Environmental Physiology Group at NTNU are working on different approaches related to the epigenetics of DCS. Changes in the physical or chemical surrounding of any organism, such as the pressure changes experienced on ascent from diving, are likely to trigger adaptive changes in gene expression patterns. Based on today's knowledge there seems to be a possible genetic link between endothelial dysfunction and DCS. Genes known to be involved in NO homeostasis display considerable individual difference in activity, which again is known to trigger susceptibility to a number of diseases. In order to examine mechanisms controlling endothelial function, we propose to examine alterations in genetic expression profiles in the endothelium in situ following decompression, using rats or mice as experimental models. By doing so we hope to gain knowledge of how specific genes respond to the physical and biochemical changes experienced in diving. Ultimately, the goal is to assess more reliable predictions for evaluating the individual risk of developing DCS, and possibly also preventing or relieving disease by means of preconditioning or pharmacological intervention.

Purposeful experimentation resulting in severe outcomes is impossible with humans, thus expansion of model capability to cover more severe cases is necessary. This will require an improved understanding of how changes in bubble location, size and profusion translate into changes in DCS severity. Collection of this information will give an ability to quantitatively scale results from animal experiments to humans. An important goal is to identify the properties of the bubble and/or host that determine progression to DCS and to use this information to prevent injury and long-term sequele.

VGE do not indicate the onset of DCS, but VGE is an indicator of decompression stress. However, since the relationship between decompression stress and DCS is not straightforward, there is no positive correlation. But there are no other measurable indications of decompression stress, and thus, measurement of VGE could be considered as the gold standard for validating decompression procedures.

It is anticipated that issues of endothelial function and NO modulation will continue to warrant strong consideration in our efforts to understand the role of VGE in the adverse effects of decompression. More profound understanding of these mechanisms will enable us to take preventive and therapeutic advantage to better predict individual risk of developing DCS, and possibly to prevent or relieve disease by means of preconditioning or pharmacological intervention.
Acknowledgments This review was supported by grants from the Central Norway Regional Health Authority and the Norwegian University of Science and Technology and by the Norwegian Petroleum Directorate, Norsk Hydro, Esso Norge and Statoil under the "Dive Contigency Contract" (No 4600002328) with Norwegian Underwater Intervention.

Open Access This article is distributed under the terms of the Creative Commons Attribution Noncommercial License which permits any noncommercial use, distribution, and reproduction in any medium, provided the original author(s) and source are credited.

\section{References}

Ahlen C, Mandal LH, Iversen OJ (1998) Identification of infectious Pseudomonas aeruginosa strains in an occupational saturation diving environment. Occup Environ Med 55:480-484

Alderton WK, Cooper CE, Knowles RG (2001) Nitric oxide synthases: structure, function and inhibition. Biochem J 357:593-615

Andric D, Petri NM, Stipancevic H, Petri LV, Kovacevic H (2003) Change of occurance of type 1 and type 2 decompression sickness of divers treated at the Croatian Naval Medical Institute in the period from 1967 to 2000. Int Marit Health 54:127-134

Balestra C, Germonpre P, Marroni A (1998) Intrathoracic pressure changes after Valsalva strain and other maneuvers: implications for divers with patent foramen ovale. Undersea Hyperb Med 25:171-174

Bergh K, Hjelde A, Iversen OJ, Brubakk AO (1993) Variability over time of complement activation induced by air bubbles in human and rabbit sera. J Appl Physiol 74:1811-1815

Bert P (1943) Barometric pressure. Researches in experimental physiology (1878). College Book Company, Colombus

Blatteau JE, Gempp E, Galland FM, Pontier JM, Sainty JM, Robinet C (2005) Aerobic exercise 2 hours before a dive to $30 \mathrm{msw}$ decreases bubble formation after decompression. Aviat Space Environ Med 76:666-669

Blatteau JE, Souraud JB, Gempp E, Boussuges A (2006) Gas nuclei, their origin, and their role in bubble formation. Aviat Space Environ Med 77:1068-1076

Blatteau JE, Gempp E, Balestra C, Mets T, Germonpre P (2008) Predive sauna and venous gas bubbles upon decompression from 400 $\mathrm{kPa}$. Aviat Space Environ Med 79:1100-1105

Boussuges A, Succo E, Juhan-Vague I, Sainty JM (1998) Activation of coagulation in decompression illness. Aviat Space Environ Med 69:129-132

Boycott AE, Damant GCC, Haldane JS (1908) The prevention of compressed air illness. J Hyg Camb 8:342-443

Boyle R (1670) New Pneumatical experiments about respiration. Philosophical Translations 5:2011-2058

Brenner I, Shephard RJ, Shek PN (1999) Immune function in hyperbaric environments, diving, and decompression. Undersea Hyperb Med 26:27-39

Brodsky SV, Zhang F, Nasjletti A, Goligorsky MS (2004) Endothelium-derived microparticles impair endothelial function in vitro. Am J Physiol 286:H1910-H1915

Broome JR, Dutka AJ, McNamee GA (1995) Exercise conditioning reduces the risk of neurologic decompression illness in swine. Undersea Hyperb Med 22:73-85

Brubakk AO (2004) Endothelium and bubble injury: the role of endothelium in decompression illness. In: Grandjean B, Méliet JL (eds) EUBS 2004. Ajaccio, Corsica

Brubakk AO, Arntzen AJ, Wienke BR, Koteng S (2003) Decompression profile and bubble formation after dives with surface decompression: experimental support for a dual phase model of decompression. Undersea Hyperb Med 30:181-193 
Brubakk AO, Duplancic D, Valic Z, Palada I, Obad A, Bakovic D, Wisloff U, Dujic Z (2005) A single air dive reduces arterial endothelial function in man. J physiol 566:901-906

Brubakk AO, Eftedal OS, Wisløff U (2007) Endothelium and diving. In: Aird W (ed) Handbook of the endothelium. Cambridge University Press, Cambridge

Carturan D, Boussuges A, Vanuxem P, Bar-Hen A, Burnet H, Gardette B (2002) Ascent rate, age, maximal oxygen uptake, adiposity, and circulating venous bubbles after diving. J Appl Physiol 93:1349-1356

Chappell M, Payne S (2005) A crevice bubble growth model for the analysis of decompression sickness. Conf Proc IEEE Eng Med Biol Soc 3:2240-2243

Chryssanthou C, Springer M, Lipschitz S (1977) Blood-brain and blood-lung barrier alteration by dysbaric exposure. Undersea Biomed Res 4:117-129

Clark RS, Carlos TM, Schiding JK, Bree M, Fireman LA, DeKosky ST, Kochanek PM (1996) Antibodies against Mac-1 attenuate neutrophil accumulation after traumatic brain injury in rats. J Neurotrauma 13:333-341

Cochran RP, Kunzelman KS, Vocelka CR, Akimoto H, Thomas R, Soltow LO, Spiess BD (1997) Perfluorocarbon emulsion in the cardiopulmonary bypass prime reduces neurologic injury. Ann Thorac Surg 63:1326-1332

Cockcroft JR (2005) Exploring vascular benefits of endothelium-derived nitric oxide. Am J Hypertens 18:177S-183S

Cross SJ, Evans SA, Thomson LF, Lee HS, Jennings KP, Shields TG (1992) Safety of subaqua diving with a patent foramen ovale. BMJ 304:481-482

Dujic Z, Duplancic D, Marinovic-Terzic I, Bakovic D, Ivancev V, Valic Z, Eterovic D, Petri NM, Wisloff U, Brubakk AO (2004) Aerobic exercise before diving reduces venous gas bubble formation in humans. J physiol 555:637-642

Dujic Z, Palada I, Obad A, Duplancic D, Bakovic D, Valic Z (2005) Exercise during a 3-min decompression stop reduces postdive venous gas bubbles. Med Sci Sports Exerc 37:1319-1323

Dujic Z, Obad A, Palada I, Ivancev V, Valic Z (2006a) Venous bubble count declines during strenuous exercise after an open sea dive to 30 m. Aviat Space Environ Med 77:592-596

Dujic Z, Palada I, Valic Z, Duplancic D, Obad A, Wisloff U, Brubakk AO (2006b) Exogenous nitric oxide and bubble formation in divers. Med Sci Sports Exerc 38:1432-1435

Dutka AJ, Kochanek PM, Hallenbeck JM (1989) Influence of granulocytopenia on canine cerebral ischemia induced by air embolism. Stroke J Cereb Circ 20:390-395

Eftedal OS (2007) Ultrasonic detection of decompression induced vascular microbubbles. Department of Ciculation and Medical Imaging NTNU, Trondheim

Eftedal OS, Lydersen S, Brubakk AO (2007a) The relationship between venous gas bubbles and adverse effects of decompression after air dives. Undersea Hyperb Med 34:99-105

Eftedal OS, Tjelmeland H, Brubakk AO (2007b) Validation of decompression procedures based on detection of venous gas bubbles: A Bayesian approach. Aviat Space Environ Med 78:94-99

Ersson A, Walles M, Ohlsson K, Ekholm A (2002) Chronic hyperbaric exposure activates proinflammatory mediators in humans. J Appl Physiol 92:2375-2380

Francis TJR, Mitchell SJ (2003) Pathophysiology of decompression sickness. In: Brubakk AO, Neuman TS (eds) Bennett and Elliott's physiology and medicine of diving. Saunders, Philadelphia, pp 530-557

Gaskins N, Vann RD, Hobbs E, Swingle M, Lee S, Needham D (2001) Surface tension and bubble formation in agar gelatin. Undersea Hyperb Med 28:56

Germonpre P, Dendale P, Unger P, Balestra C (1998) Patent foramen ovale and decompression sickness in sports divers. J Appl Physiol $84: 1622-1626$
Germonpre P, Hastir F, Dendale P, Marroni A, Nguyen AF, Balestra C (2005) Evidence for increasing patency of the foramen ovale in divers. Am J Cardiol 95:912-915

Germonpre P, Pontier JM, Gempp E, Blatteau JE, Deneweth S, Lafere P, Marroni A, Balestra C (2009) Pre-dive vibration effect on bubble formation after a 30-m dive requiring a decompression stop. Aviat Space Environ Med 80:1044-1048

Glavas D, Markotic A, Valic Z, Kovacic N, Palada I, Martinic R, Breskovic T, Bakovic D, Brubakk AO, Dujic Z (2008) Expression of endothelial selectin ligands on human leukocytes following dive. Exp Biol Med (Maywood, NJ) 233:1181-1188

Gonzalez MA, Selwyn AP (2003) Endothelial function, inflammation, and prognosis in cardiovascular disease. Am J Med 8:99s-106s

Green D, Cheetham C, Henderson C, Weerasooriya R, O'Driscoll G (2002) Effect of cardiac pacing on forearm vascular responses and nitric oxide function. Am J Physiol 283:H1354-H1360

Gutvik CR, Brubakk AO (2008) A dynamic 2-phase model for vascular bubble formation during decompression of divers. IEEE Trans Biomed Eng (in press)

Hallenbeck JM, Bove AA, Elliott DH (1975) Mechanisms underlying spinal cord damage in decompression sickness. Neurology 25:308-316

Haram PM, Adams V, Kemi OJ, Brubakk AO, Hambrecht R, Ellingsen O, Wisloff U (2006) Time-course of endothelial adaptation following acute and regular exercise. Eur J Cardiovasc Prev Rehabil 13:585-591

Havnes MB, Hjelde A, Brubakk AO, Møllerløkken A (2010) S100B and its relation to intravascular bubbles following decompression. Diving Hyperb Med 40:4

Helps SC, Gorman DF (1991) Air embolism of the brain in rabbits pretreated with mechlorethamine. Stroke J Cereb Circ 22:351-354

Hjelde A, Bolstad G, Brubakk AO (2002) The effect of air bubbles on rabbit blood brain barrier. Undersea Hyperb Med 29:31-38

Huang KL, Wu CP, Chen YL, Kang BH, Lin YC (2003) Heat stress attenuates air bubble-induced acute lung injury: a novel mechanism of diving acclimatization. J Appl Physiol 94:1485-1490

Jimenez JJ, Jy W, Mauro LM, Soderland C, Horstman LL, Ahn YS (2003) Endothelial cells release phenotypically and quantitatively distinct microparticles in activation and apoptosis. Thromb Res 109:175-180

Kavdia M, Popel AS (2004) Contribution of nNOS- and eNOS-derived NO to microvascular smooth muscle NO exposure. J Appl Physiol 97:293-301

Kerem M, Mehmet C (2000) Endothelial adhesivity, pulmonary hemodynamics and nitric oxide synthesis in ischemia-reperfusion. Eur J Cardiothorac Surg 18:248-252

Kingwell BA, Sherrard B, Jennings GL, Dart AM (1997) Four weeks of cycle training increases basal production of nitric oxide from the forearm. Am J Physiol 272:H1070-H1077

Klibanov AL (2006) Microbubble contrast agents: targeted ultrasound imaging and ultrasound-assisted drug-delivery applications. Invest Radiol 41:354-362

Kregel KC (2002) Heat shock proteins: modifying factors in physiological stress responses and acquired thermotolerance. J Appl Physiol 92:2177-2186

Langton P (1996) Patent foramen ovale in underwater medicine. SPUMS J 26:186-191

Liebermann L (1957) Air bubbles in water. J Appl Physics 28:205-211

Lippmann J, Mitchell S (2005) Deeper into Diving. J.L. Publications, Melbourne

Ljubkovic M, Dujic Z, Mollerlokken A, Bakovic D, Obad A, Breskovic T, Brubakk AO (2010a) Venous and arterial bubbles at rest after no-decompression air dives. Med Sci Sports Exerc [Epub ahead of print]

Ljubkovic M, Marinovic J, Obad A, Breskovic T, Gaustad SE, Dujic Z (2010b) High incidence of venous and arterial gas emboli at rest 
after trimix diving without protocol violations. J Appl Physiol 109:1670-1674

Lutz J, Herrmann G (1984) Perfluorochemicals as a treatment of decompression sickness in rats. Pflugers Arch 410:174-177

Mahon RT, Dainer HM, Nelson JW (2006) Decompression sickness in a swine model: isobaric denitrogenation and perfluorocarbon at depth. Aviat Space Environ Med 77:8-12

Martin JD, Thom SR (2002) Vascular leukocyte sequestration in decompression sickness and prophylactic hyperbaric oxygen therapy in rats. Aviat Space Environ Med 73:565-569

Mollerlokken A, Berge VJ, Jorgensen A, Wisloff U, Brubakk AO (2006) Effect of a short-acting NO donor on bubble formation from a saturation dive in pigs. J Appl Physiol 101:1541-1545

Moncada S, Higgs EA (2006) Nitric oxide and the vascular endothelium. Handbook of experimental pharmacology: 213-254

Montcalm-Smith E, Caviness J, Chen Y, McCarron RM (2007) Stress biomarkers in a rat model of decompression sickness. Aviat Space Environ Med 78:87-93

Moon RE (2004) Treatment of decompression illness. In: Bove AA (ed) Bove and Davis' diving medicine. Saunders, Philadelphia, pp 195-225

Moon RE, Camporesi EM, Kisslo JA (1989) Patent foramen ovale and decompression sickness in divers. Lancet 1:513-514

Moon RE, Vann RD, Bennett PB (1995) The physiology of decompression illness. Sci Am 273:70-77

Neuman TS (2003) Arterial gas embolism and pulmonary barotrauma. In: Brubakk AO, Neuman TS (eds) Bennett and Elliott's physiology and medicine of diving. Saunders, Philadelphia, pp 557-577

Nishi RY, Brubakk AO, Eftedal OS (2003) Bubble detection. In: Brubakk AO, Neuman TS (eds) Bennett and Elliott's physiology and medicine of diving. Saunders, Philadelphia, pp 501-530

Nossum V, Koteng S, Brubakk AO (1999) Endothelial damage by bubbles in the pulmonary artery of the pig. Undersea Hyperb Med 26:1-8

Nossum V, Hjelde A, Brubakk AO (2002) Small amounts of venous gas embolism cause delayed impairment of endothelial function and increase polymorphonuclear neutrophil infiltration. Eur J Appl Physiol 86:209-214

Nossum V, Hjelde A, Bergh K, Ustad AL, Brubakk AO (2003) AntiC5a monoclonal antibodies and pulmonary polymorphonuclear leukocyte infiltration-endothelial dysfunction by venous gas embolism. Eur J Appl Physiol 89:243-248

Nyquist PA, Dick EJ Jr, Buttolph TB (2004) Detection of leukocyte activation in pigs with neurologic decompression sickness. Aviat Space Environ Med 75:211-214

Obad A, Marinovic J, Ljubkovic M, Breskovic T, Modun D, Boban M, Dujic Z (2010) Successive deep dives impair endothelial function and enhance oxidative stress in man. Clin Physiol Funct Imaging 30:432-438

Philp RB, Inwood MJ, Warren BA (1972) Interactions between gas bubbles and components of the blood: implications in decompression sickness. Aerosp Med 43:946-953

Rosenblum WI (1997) Tetrahydrobiopterine, a cofactor for nitric oxide synthase, produces endothelium-dependent dilation of mouse pial arterioles. Stroke J Cereb Circ 28(1):186-189

Sawatzky KD (1991) The relationship between intravascular Dopplerdetected gas bubbles and decompression sickness after bounce diving in humans. York University, Toronto

Schoettle RJ, Kochanek PM, Magargee MJ, Uhl MW, Nemoto EM (1990) Early polymorphonuclear leukocyte accumulation correlates with the development of posttraumatic cerebral edema in rats. J Neurotrauma 7:207-217

Shaul PW (2002) Regulation of endothelial nitric oxide synthase: location, location, location. Annu Rev Physiol 64:749-774

Smith DJ, Francis TJ, Hodgson M, Murrison AW, Sykes JJ (1990) Interatrial shunts and decompression sickness in divers. Lancet 335:914-915
Spahn DR (2000) Current status of artificial oxygen carriers. Adv Drug Deliv Rev 40:143-151

Sparacia G, Banco A, Sparacia B, Midiri M, Brancatelli G, Accardi M, Lagalla R (1997) Magnetic resonance findings in scuba divingrelated spinal cord decompression sickness. MAGMA 5:111-115

Talu E, Hettiarachchi K, Zhao S, Powell RL, Lee AP, Longo ML, Dayton PA (2007) Tailoring the size distribution of ultrasound contrast agents: possible method for improving sensitivity in molecular imaging. Mol Imaging 6:384-392

Thom SR, Yang M, Bhopale VM, Huang S, Milovanova TN (2011) Microparticles initiate decompression-induced neutrophil activation and subsequent vascular injuries. J Appl Physiol 110:340 351

Tikuisis P, Gerth WA (2003) Decompression Theory. In: Brubakk AO, Neuman TS (eds) Bennett and Elliott's physiology and medicine of diving. Saunders, Philadelphia, pp. 419-455

Triggle CR, Hollenberg M, Anderson TJ, Ding H, Jiang Y, Ceroni L, Wiehler WB, Ng ES, Ellis A, Andrews K, McGuire JJ, Pannirselvam M (2003) The endothelium in health and disease-a target for therapeutic intervention. J Smooth Muscle Res 39:249-267

Tuman KJ, Spiess BD, McCarthy RJ, Ivankovich AD (1986) Cardiorespiratory effects of venous air embolism in dogs receiving a perfluorocarbon emulsion. J Neurosurg 65:238-244

van Hulst RA, Klein J, Lachmann B (2003) Gas embolism: pathophysiology and treatment. Clin Physiol Funct Imaging 23:237-246

VanWijk MJ, VanBavel E, Sturk A, Nieuwland R (2003) Microparticles in cardiovascular diseases. Cardiovasc Res 59:277-287

Vik A, Jenssen BM, Brubakk AO (1993) Arterial gas bubbles after decompression in pigs with patent foramen ovale. Undersea Hyperb Med 20:121-131

Vince RV, Chrismas B, Midgley AW, McNaughton LR, Madden LA (2009) Hypoxia mediated release of endothelial microparticles and increased association of S100A12 with circulating neutrophils. Oxid Med Cell Longev 2:2-6

Ward CA, McCullough D, Fraser WD (1987) Relation between complement activation and susceptibility to decompression sickness. J Appl Physiol 62:1160-1166

Wilmshurst P, Bryson P (2000) Relationship between the clinical features of neurological decompression illness and its causes. Clin Sci (Lond) 99:65-75

Wilmshurst P, Byrne C, Webb-Peploe MM (1989) Relation between interatrial shunts and decompression sickness in divers. Lancet 2(8675):1302-1306

Wilmshurst P, Davidson C, O’Connell G, Byrne C (1994) Role of cardiorespiratory abnormalities, smoking and dive characteristics in the manifestations of neurological decompression illness. Clin Sci (Lond) 86:297-303

Wisloff U, Richardson RS, Brubakk AO (2003) NOS inhibition increases bubble formation and reduces survival in sedentary but not exercised rats. J Physiol 546:577-582

Wisloff U, Richardson RS, Brubakk AO (2004) Exercise and nitric oxide prevent bubble formation: a novel approach to the prevention of decompression sickness? J Physiol 555:825-829

Wisloff U, Haram PM, Brubakk AO (2007) Exercise and the Endothelium. In: Aird W (ed) Endothelial Biomedicine. Cambrigde University Press, Cambridge, pp 506-515

Yount DE (1988) Growth of bubbles from nuclei. In: Brubakk AO, Hemmingsen BB, Sundnes G (eds) Supersaturation and Bubble Formation in Fluids and Organisms. Tapir Publishers, Trondheim, pp 131-164

Zheng Q, Durben DJ, Wolf GH, Angell CA (1991) Liquids at large negative pressures: water at the homogenous nucleation limit. Science 254:829-832 